\title{
Unraveling the Hydroxide Ion Transportation Mechanism Along the Surface of Two-Dimensional Mg-Al Layered Double Hydroxide
}

\author{
Nanosheets
}

\section{Supporting Information}

\author{
Le Shi*†, Zhixuan Ying $^{\dagger}$, Ao Xu*, Yonghong Cheng ${ }^{\dagger}$
}

$\uparrow$ State Key Laboratory of Electrical Insulation and Power Equipment, Center of Nanomaterials for Renewable Energy, School of Electrical Engineering, Xi'an Jiaotong University, Xi'an 710049, China

\$ School of Aeronautics, Northwestern Polytechnical University, Xi’an 710072, China

\section{Water density distribution}

For MD simulations, the trajectories were recorded every $100 \mathrm{MD}$ steps. For AIMD simulations, the trajectories were recorded every $1 \mathrm{MD}$ step. The water density distribution $\rho(O)$ was calculated as:

$$
\rho(O)=\frac{M \sum_{i=1}^{N_{\text {frame }}} n_{O i}}{N_{A} A B \Delta z N_{\text {frame }}}
$$

Where $M$ is the mass for 1 mole water, $N_{\text {frame }}$ is the number of frames, $n_{O i}$ is the number of oxygen atoms found inside the slice at frame $i, N_{A}$ is the Avogadro constant, $A$ and $B$ are the cell dimensions at $x$ and $y$ directions, and $\Delta z$ is set to be $0.2 \AA$. The water density distribution $\rho(H)$ was calculated as

$$
\rho(H)=\frac{M \sum_{i=1}^{N_{\text {frame }}} n_{H i}}{2 N_{A} A B \Delta z N_{\text {frame }}}
$$

Where $n_{H i}$ is the number of hydrogen atoms found inside the slice at frame $i$.

\footnotetext{
${ }^{*}$ Corresponding Author. Email: le.shi@mail.xjtu.edu.cn (Le Shi)
} 


\section{H-bond correlation function}

The hydrogen bonds were identified using a set of geometric criteria [S1]. Two molecules are considered to form a hydrogen bond if the distance between the oxygen atoms of these molecules $\left(\mathrm{R}_{\mathrm{O}-\mathrm{O}}\right)$ is smaller than $3.3 \AA$ or the distance between the oxygen and chloride atoms of these molecules $\left(\mathrm{R}_{\mathrm{O}-\mathrm{Cl}}\right)$ is smaller than $3.8 \AA$, and the same time the angle between the O---O or O--- $\mathrm{Cl}$ and the $\mathrm{O}---\mathrm{H}$ vector is less than $30^{\circ}$. The cutoff value of $\mathrm{R}_{\mathrm{O}-\mathrm{O}}$ and $\mathrm{R}_{\mathrm{O}-\mathrm{Cl}}$ are determined based on the radial distribution function obtained from AIMD simulations, as shown in Fig. S14.

We defined two variables $h(t)$ and $H(t)$ to characterize the hydrogen bond dynamics. $h(t)$ is equal to 1 if a particular water pair is hydrogen bonded and zero otherwise. $H(t)=1$ indicates that a pair of water molecules remains hydrogen bonded from $\mathrm{t}=0$ to time $\mathrm{t}$ and zero otherwise. The hydrogen bond time correlation function $S_{H B}(t)$ is defined as [S2]:

$$
S_{H B}(t)=\frac{<h(0) H(t)>}{<h(0)^{2}>}
$$

It gives the probability that an initially hydrogen bonded pair remains hydrogen bonded all the time from $t=0$ to $t$.

\section{Dipole correlation function}

The dipole correlation function characterized the rotational motion of water molecules as [S3]:

$$
\Gamma=\frac{<\boldsymbol{\mu}(0) \boldsymbol{\mu}(t)>}{<\boldsymbol{\mu}(0)^{2}>}
$$

Where $\boldsymbol{\mu}(t)$ is the dipole vector of a water molecule at time $t$, which was calculated by assigning a charge of -2 to the oxygen atom and charges of +1 to the hydrogen (deuterium) atoms.

\section{Construction of S system}

The S system was constructed by removing water atoms from L system. At first, water molecules in the IM region of $\mathrm{L}$ system were removed, and the system went through a $2 \mathrm{~ns}$ MD simulation in NPT ensemble ( $T=300 \mathrm{~K}, P=1 \mathrm{~atm})$. Afterwards, the water molecules were removed one by one, followed by a series of $2 \mathrm{~ns}$ MD simulations in 
NPT ( $T=300 \mathrm{~K}, P=1 \mathrm{~atm}$ ) ensemble until the height of the system approaches an experimental value of $1.1 \mathrm{~nm}$ [S4]. Then $2 \mathrm{~ns}$ MD simulation in NVT (T=300 K) ensemble and 20 ps AIMD simulation in NVT ensemble were performed to equilibrate the system.

\section{Calculation of diffusion coefficient}

For bulk water system and the $42 \mathrm{water}+\mathrm{Cl}^{-}$system, the mean square displacement was calculated as:

$$
\left.\operatorname{MSD}=\langle| \boldsymbol{r}(t)-\boldsymbol{r}(0)]^{2}\right\rangle
$$

And the isotropic diffusion coefficient was calculated as

$$
D=\frac{1}{6} \lim _{t \rightarrow \infty} \frac{\left.\langle| \boldsymbol{r}(t)-\boldsymbol{r}(0)]^{2}\right\rangle}{\Delta t}
$$

For L and S systems, the mean square displacement along $x y$ direction was calculated as:

$$
\left.\left.\operatorname{MSD}=\langle| \boldsymbol{r}_{x y}(t)-\boldsymbol{r}_{x y}(0)\right]^{2}\right\rangle
$$

And the lateral diffusion coefficient was calculated as:

$$
D=\frac{1}{4} \lim _{t \rightarrow \infty} \frac{\left.\left.\langle| \boldsymbol{r}_{x y}(t)-\boldsymbol{r}_{x y}(0)\right]^{2}\right\rangle}{\Delta t}
$$

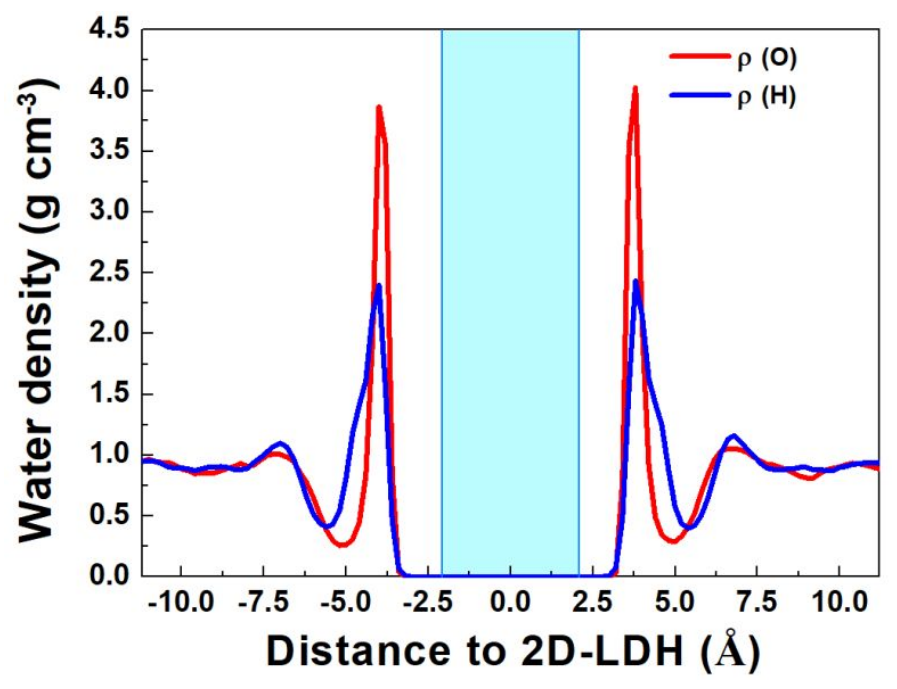


Figure S1. Density profile of water as a function of distance to 2D-LDH of L system. The data was obtained from a $1 \mathrm{~ns}$ MD simulation. The light blue area represents the 2D-LDH.
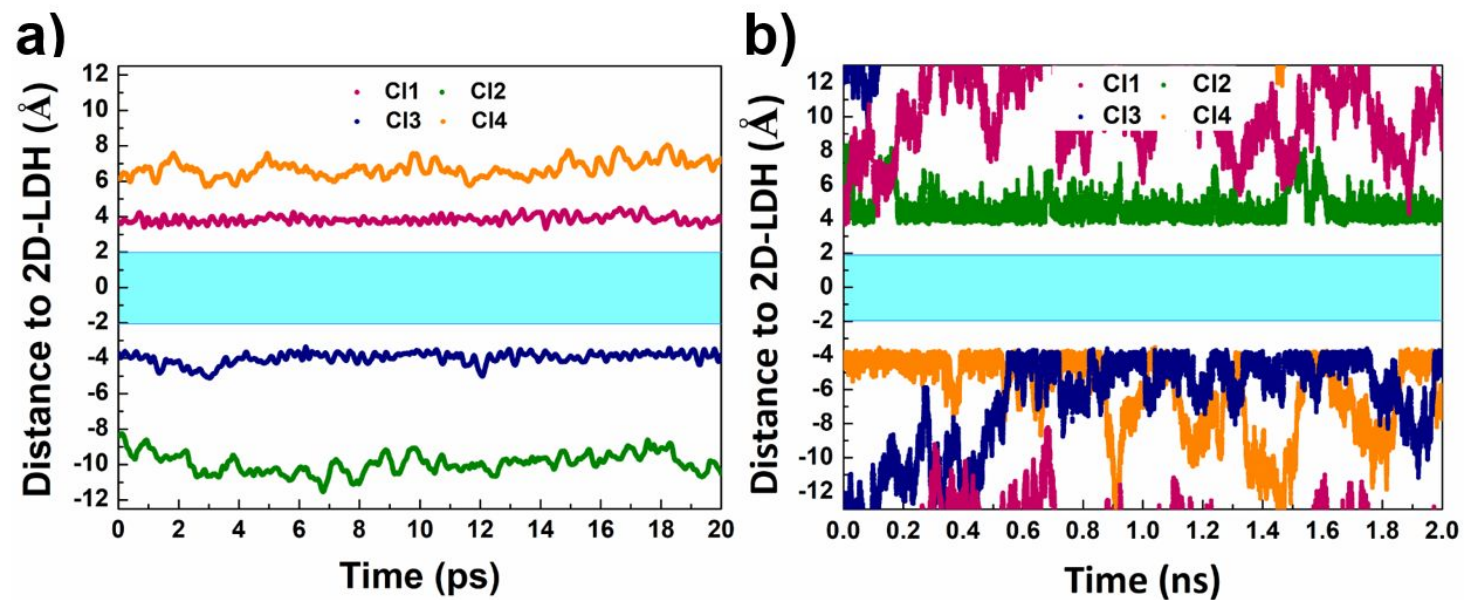

Figure S2. Trajectories of $\mathrm{Cl}^{-}$ions in L system (a) AIMD simulation (b) MD simulation. The light blue area represents the 2D-LDH. 


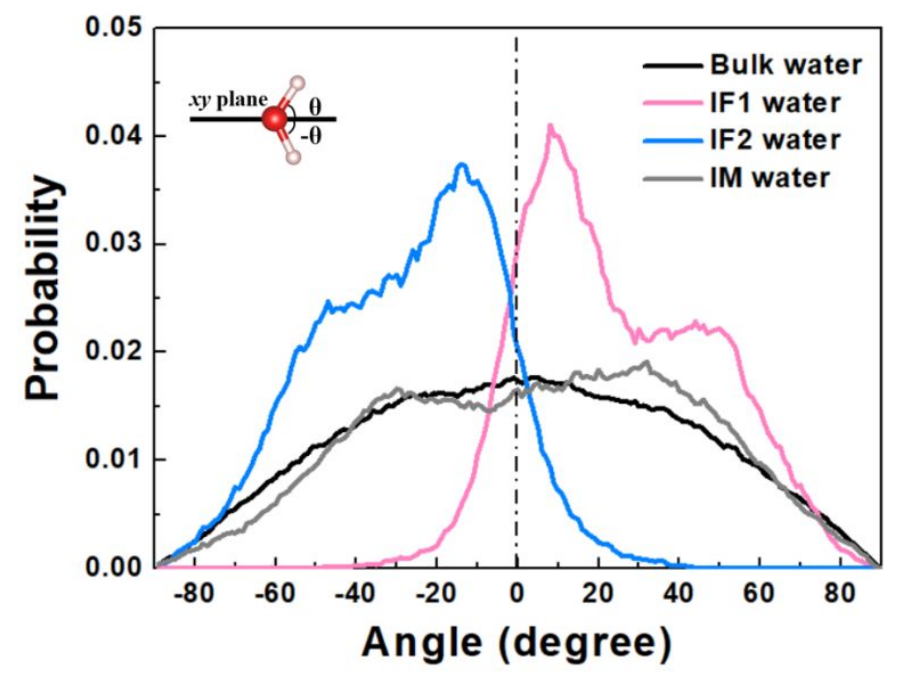

Figure S3. Distribution of the angle formed between $\mathrm{OH}$ bond and the $x y$-plane in different regions of $\mathrm{L}$ system. The data were collected from a $20 \mathrm{ps}$ AIMD simulation.

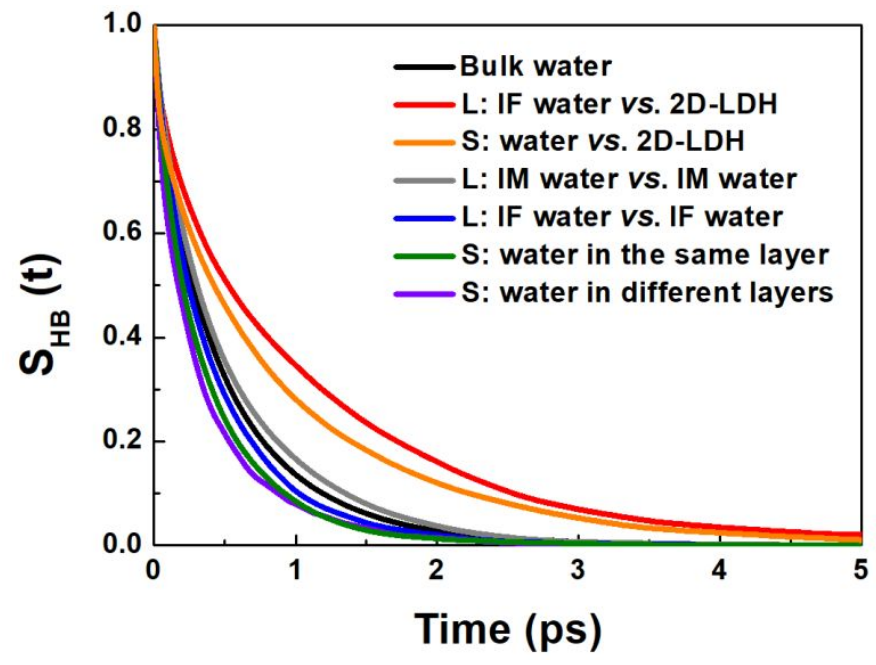

Figure S4. Time dependence of the continuous hydrogen bond correlation functions for different types of hydrogen bonds. The data were collected from AIMD simulations. 


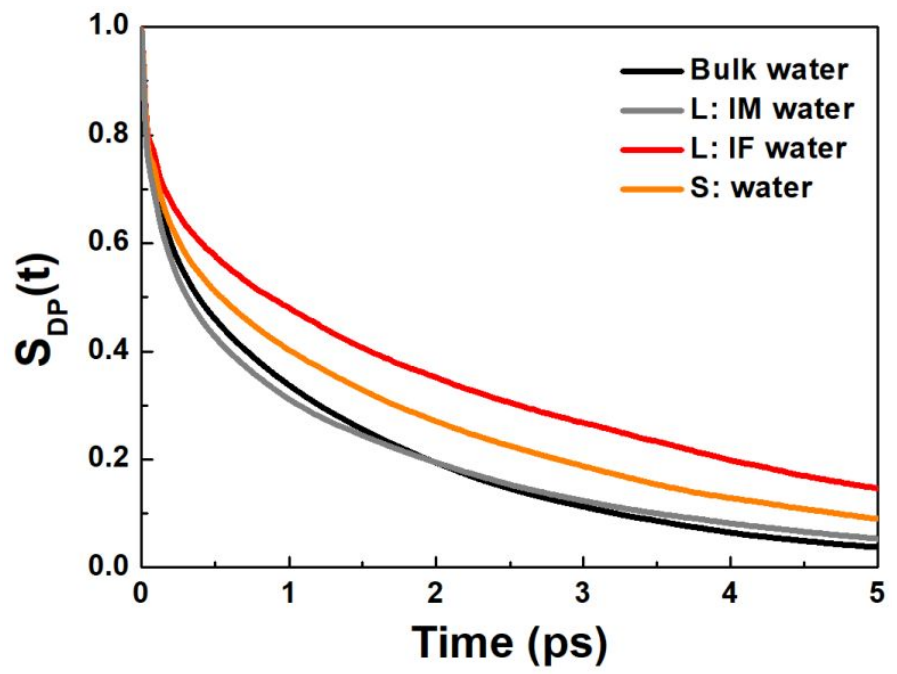

Figure S5. Time dependence of the dipole correlation functions for water molecules in different systems. The data were collected from AIMD simulations.

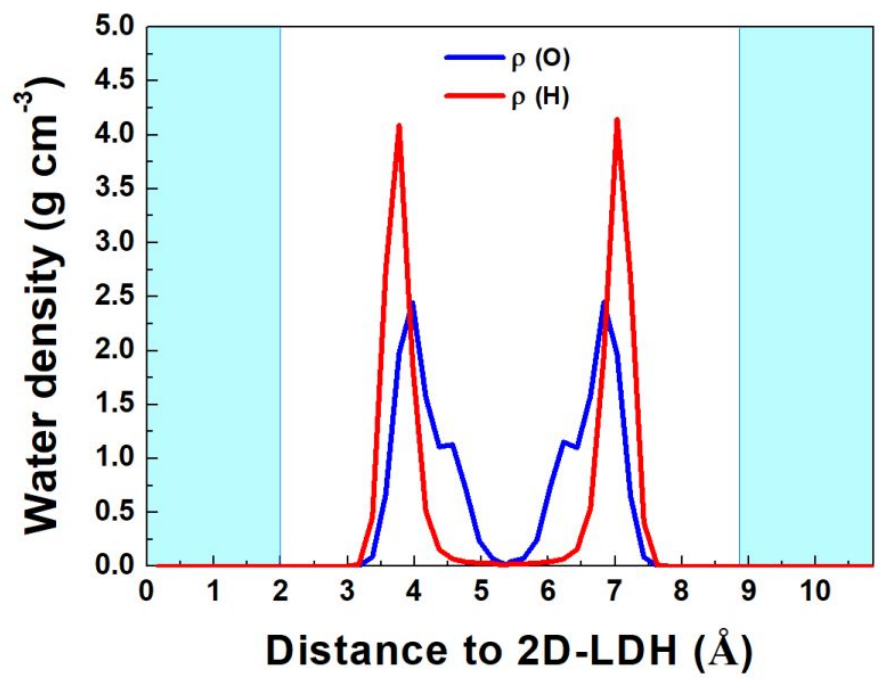

Figure S6. Density profile of water as a function of distance to 2D-LDH of S system.

The data were collected from a $1 \mathrm{~ns}$ MD simulation. The light blue area represents the 2D-LDH. 

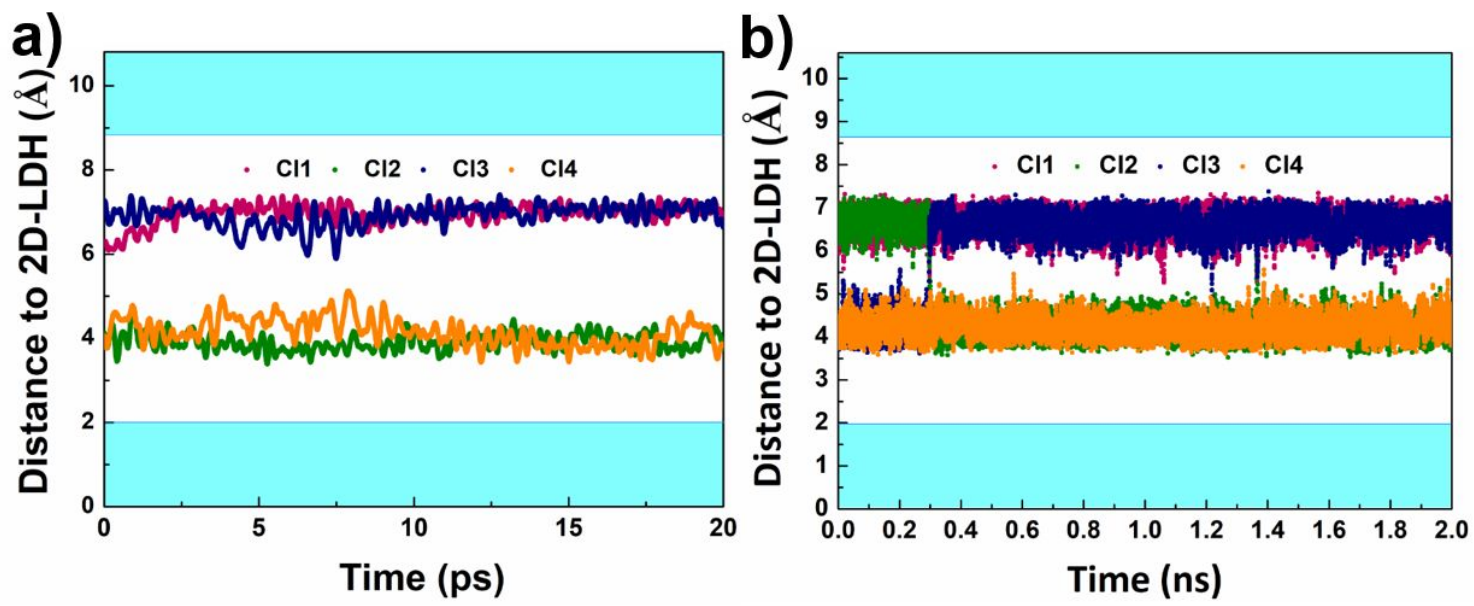

Figure S7. Trajectories of $\mathrm{Cl}^{-}$ions in S system (a) AIMD simulation (b) MD simulation.

The light blue area represents the 2D-LDH.

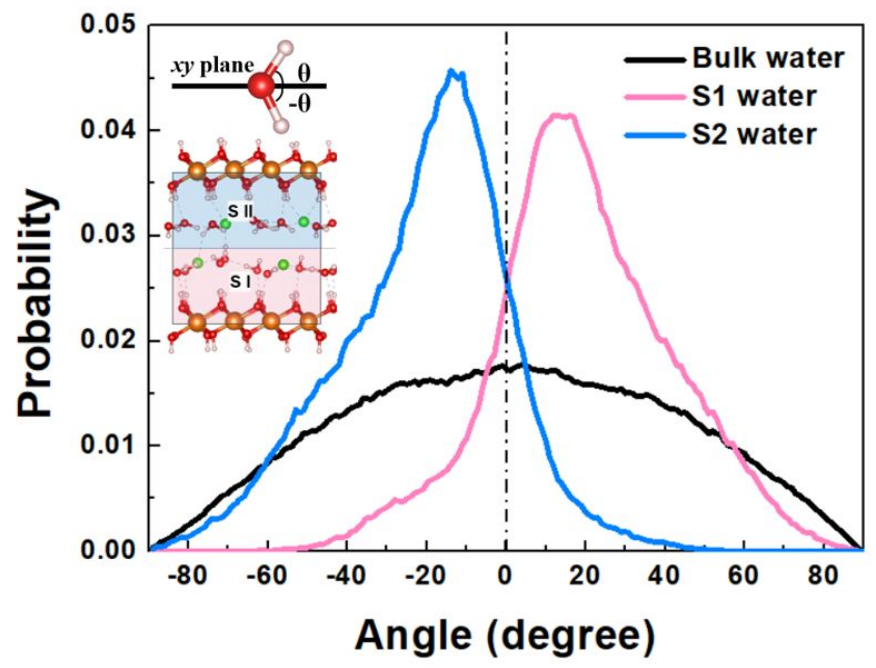

Figure S8. Distribution of the angle formed between $\mathrm{OH}$ bond and the $x y$-plane in different regions of S system. The data was collected from a 20 ps AIMD simulation. 


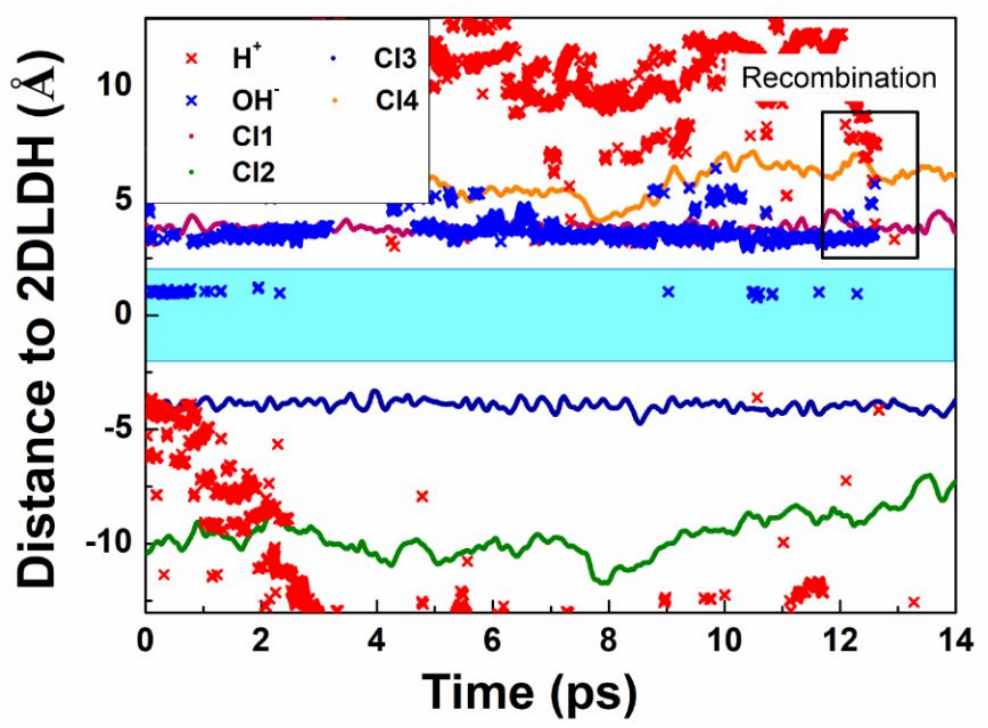

Figure S9. Proton and hydroxide ion diffusion trajectory in L system before recombination occur.

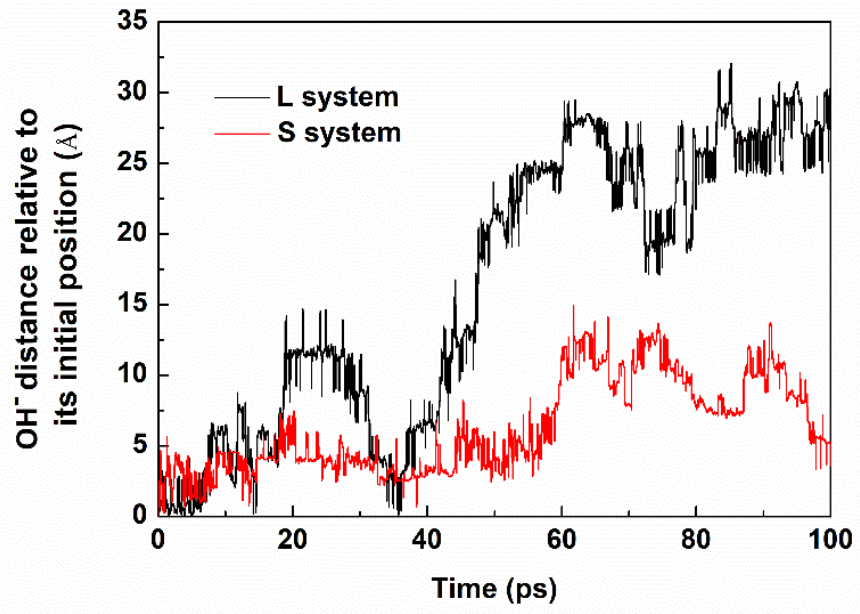

Figure S10. The distance of $\mathrm{OH}$ - relative to its initial position as a function of time in both $\mathrm{L}$ and $\mathrm{S}$ systems. 


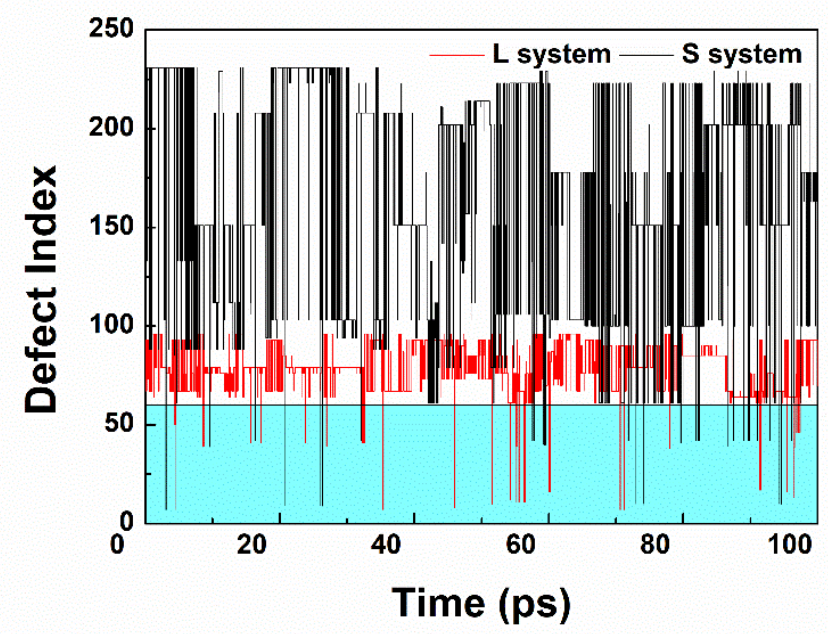

Figure S11. The index of oxygen atom that carries the charge of $\mathrm{OH}-$ as a function of time. The blue area indicates the oxygen atoms that belongs to the 2D-LDH slab.
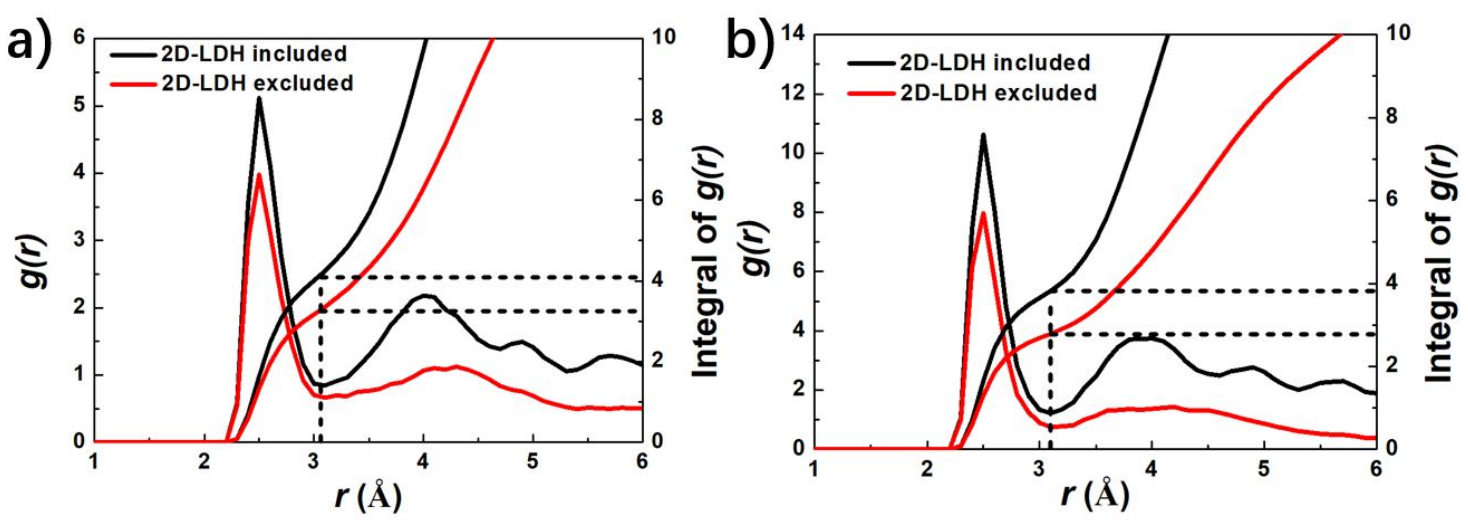

Figure S12. Radial distribution functions of oxygen atoms and the hydroxide oxygen $\mathrm{O}^{*}$ in different systems. (a) L system (b) S system. The data were collected from AIMD simulations. 


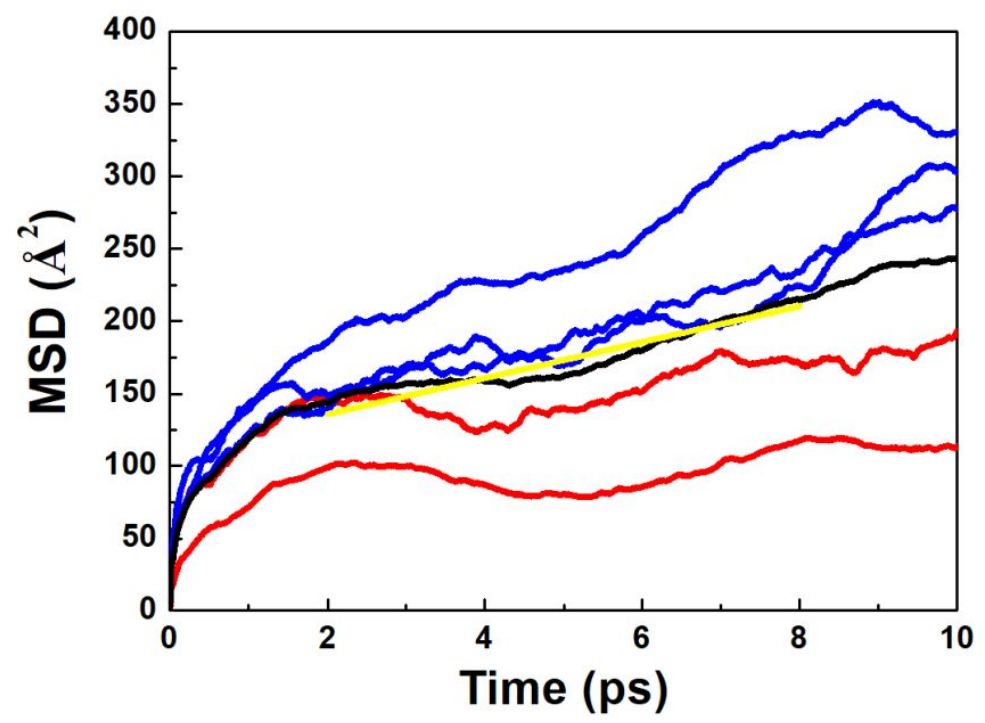

Figure S13. Mean square displacement of $\mathrm{OH}^{-}$in bulk water system from various trajectory realizations. The blue curves are trajectory realizations yielding an effective higher diffusion constant while the red curves are those leading to a lower diffusion constant. The black curve is the average of all the realizations. The yellow dashed line represents the linear region used to obtain the diffusion constant.

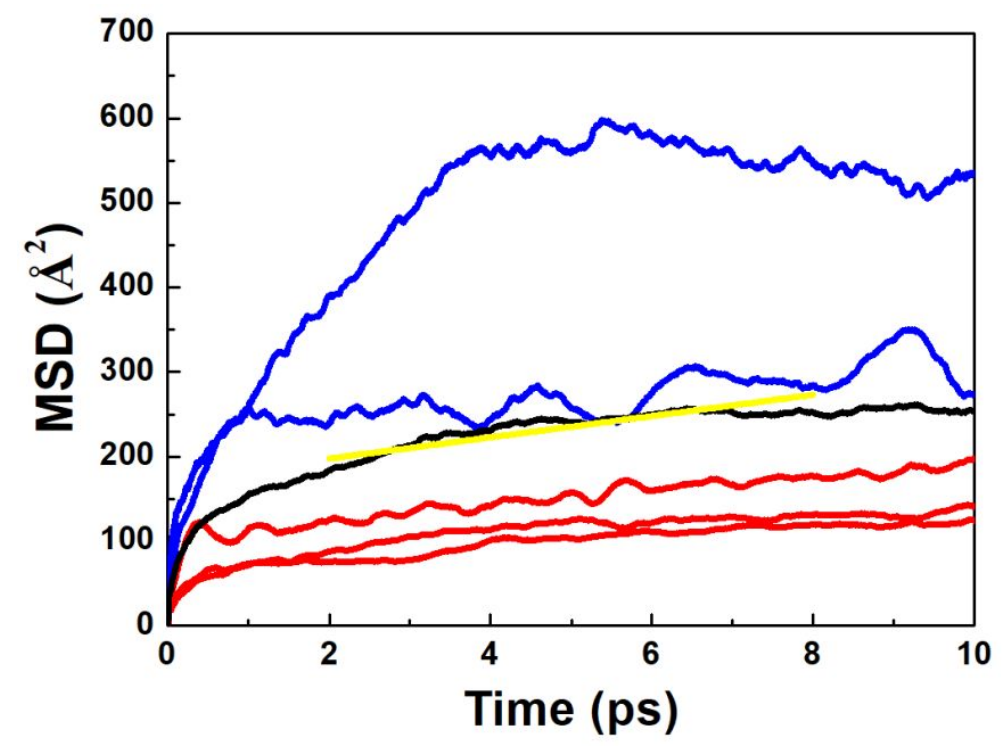

Figure S14. Mean square displacement of $\mathrm{OH}^{-}$in 42 water $+\mathrm{Cl}^{-}$system from various trajectory realizations. The blue curves are trajectory realizations yielding an effective higher diffusion constant while the red curves are those leading to a lower diffusion constant. The black curve is the average of all the realizations. The yellow dashed line represents the linear region used to obtain the diffusion constant. 


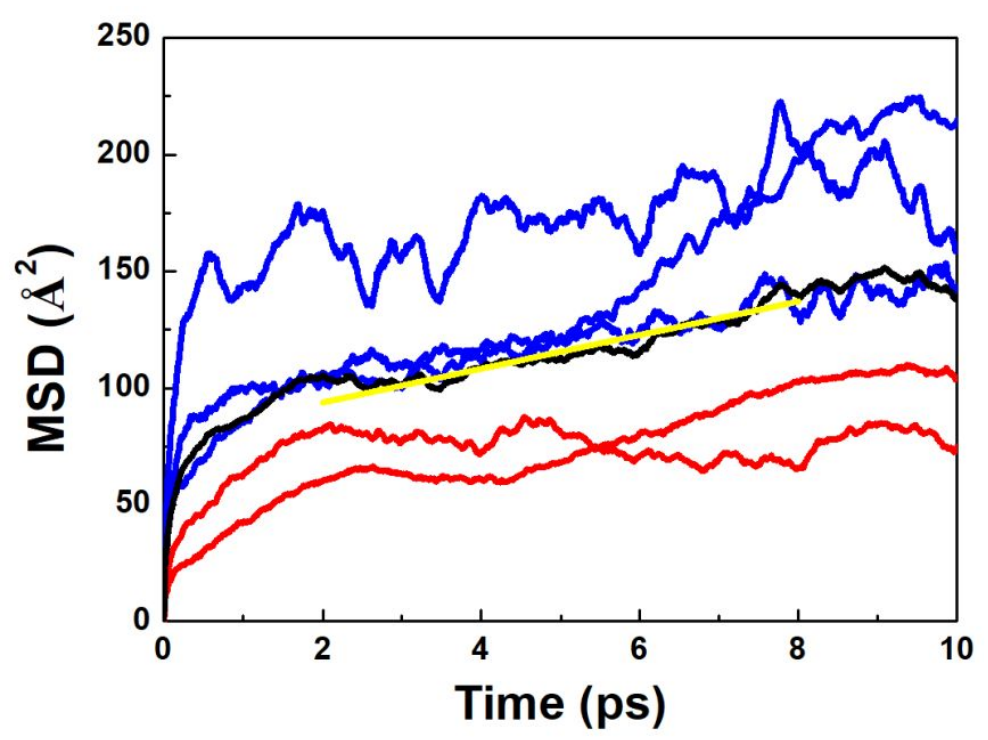

Figure S15. Mean square displacement of $\mathrm{OH}^{-}$along the $x y$ surface in L system from various trajectory realizations. The blue curves are trajectory realizations yielding an effective higher diffusion constant while the red curves are those leading to a lower diffusion constant. The black curve is the average of all the realizations. The yellow dashed line represents the linear region used to obtain the diffusion constant.

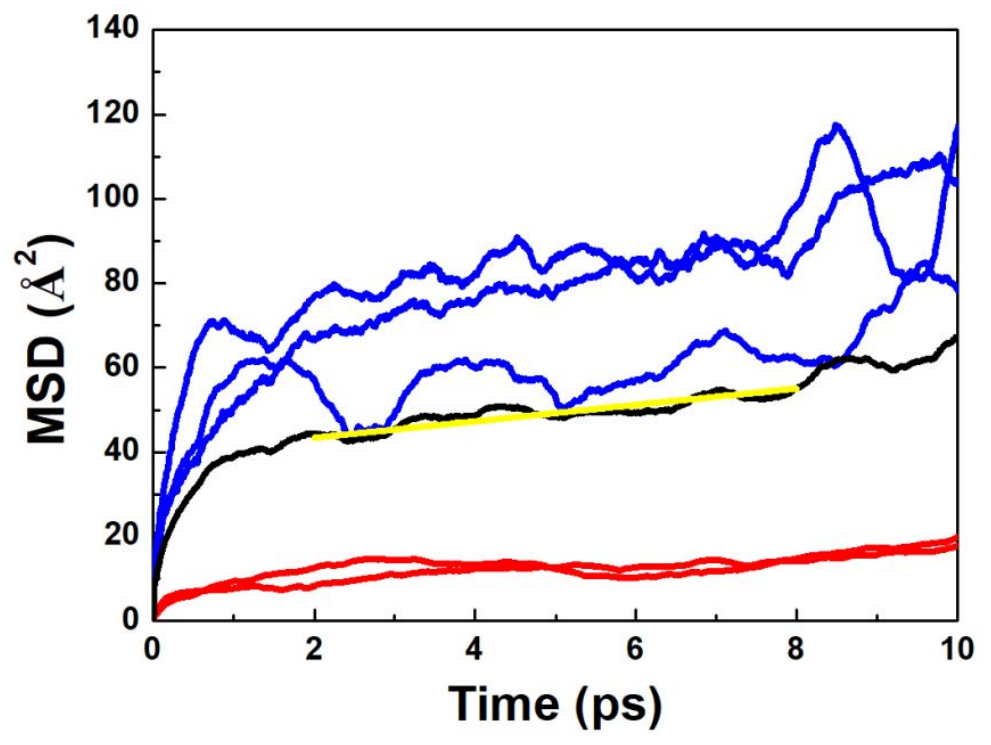

Figure S16. Mean square displacement of $\mathrm{OH}^{-}$along the $x y$ surface in S system from various trajectory realizations. The blue curves are trajectory realizations yielding an effective higher diffusion constant while the red curves are those leading to a lower 
diffusion constant. The black curve is the average of all the realizations. The yellow dashed line represents the linear region used to obtain the diffusion constant.

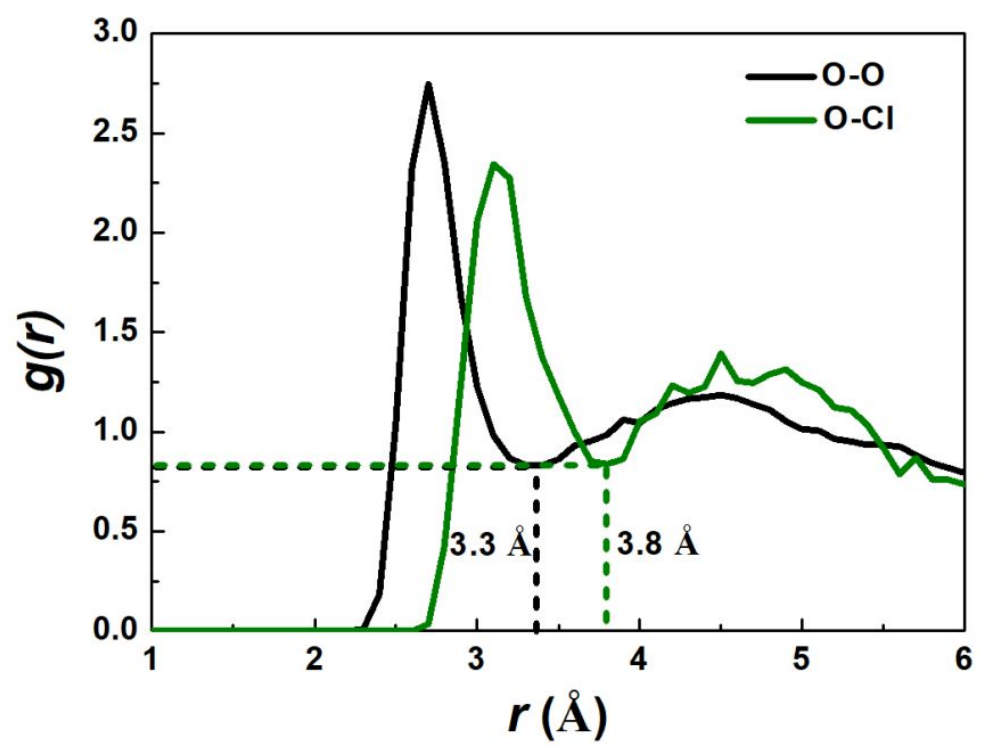

Figure S17. oxygen-oxygen and oxygen-chloride radial distribution function in the 42 water $+\mathrm{Cl}^{-}$system. The data were collected from a 20 ps AIMD simulation.
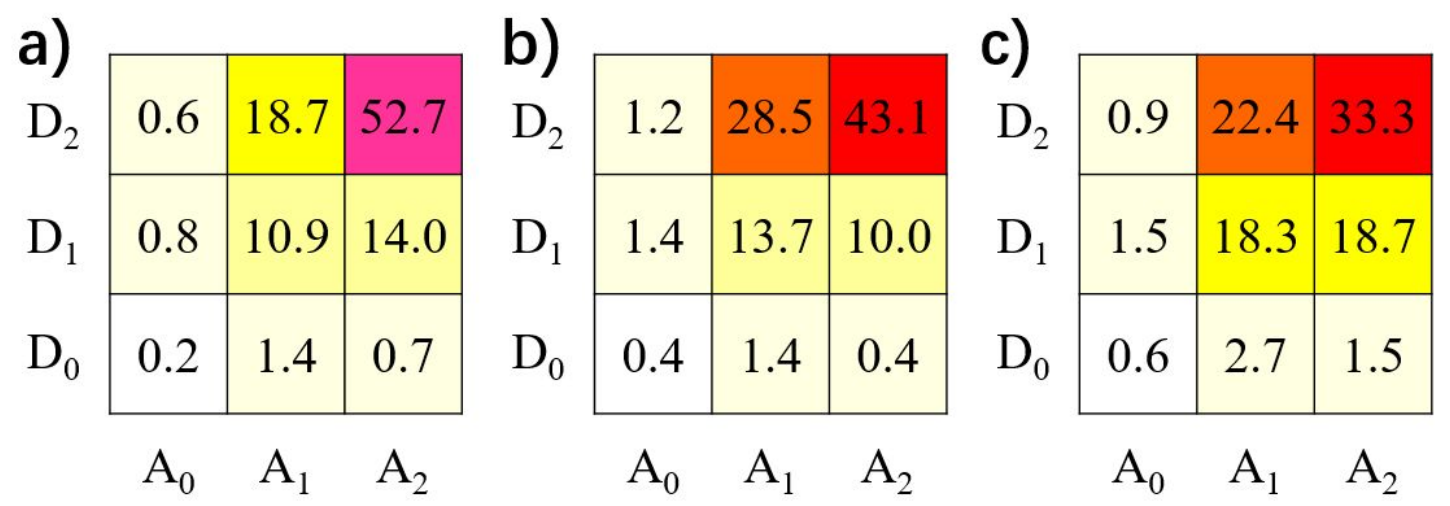

Figure S18. Hydrogen bond statistics of water molecules in different systems. (a) Bulk water b) 42 water $+\mathrm{Cl}^{-}$system (only hydrogen bonds formed between water molecules were considered c) 42 water $+\mathrm{Cl}^{-}$system (all hydrogen bonds were considered). The digital numbers in the square represent percentage (\%) of corresponding water 
molecules that accept and/or donate $i=2,1$, or $0 \mathrm{H}$-bonds denoted by $\mathrm{A}_{\mathrm{i}}$ and $\mathrm{D}_{\mathrm{i}}$, respectively. The data were collected from AIMD simulations.
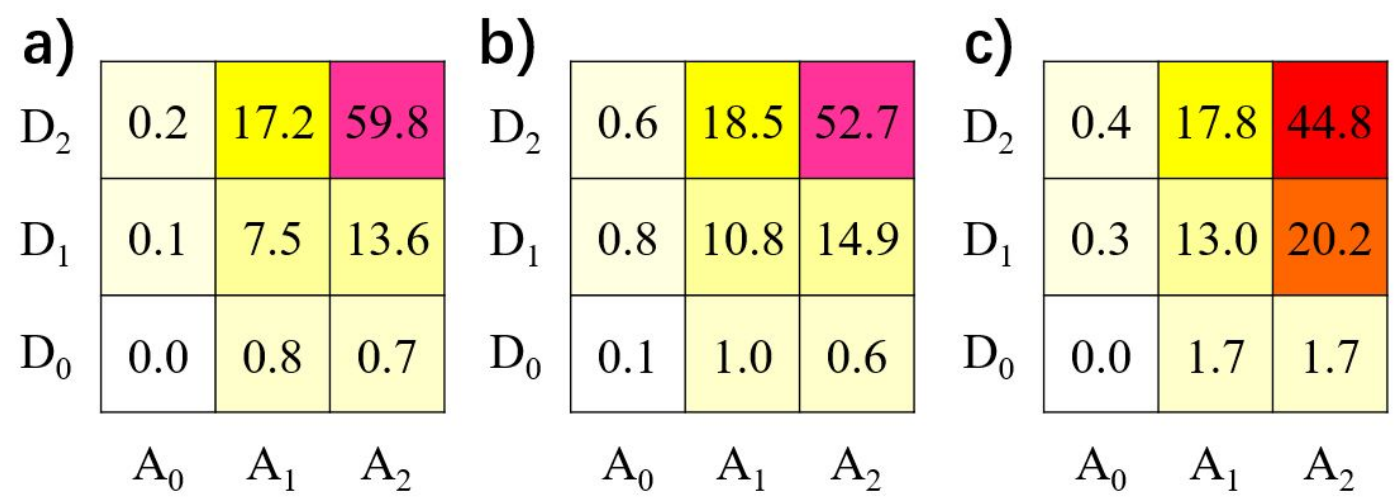

Figure S19. Hydrogen bond statistics of water molecules in different systems. (a) IF region in L system (b) IM region in L system (c) S system. All kinds of hydrogen bonds were considered. The digital numbers in the square represent percentage $(\%)$ of corresponding water molecules that accept and/or donate $i=2,1$, or $0 \mathrm{H}$-bonds denoted by $A_{i}$ and $D_{i}$, respectively. The data was collected from AIMD simulations.

a)

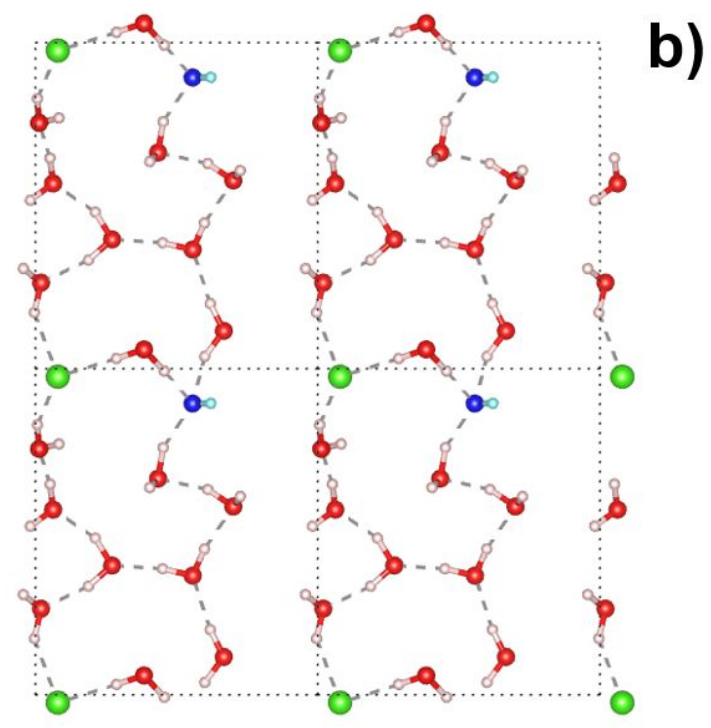

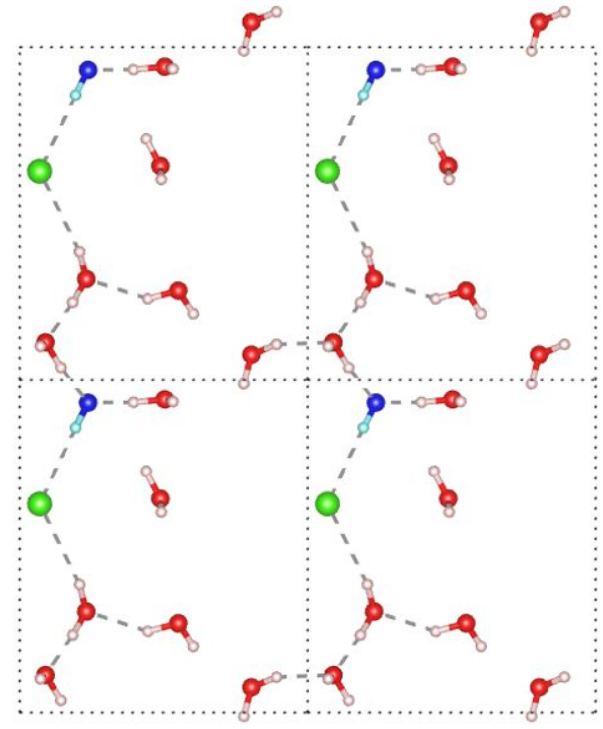

Figure S20. Snapshots of the hydrogen bonding network formed by the first water layer near 2D-LDH in different systems in AIMD simulations. (a) L system (b) S system. 


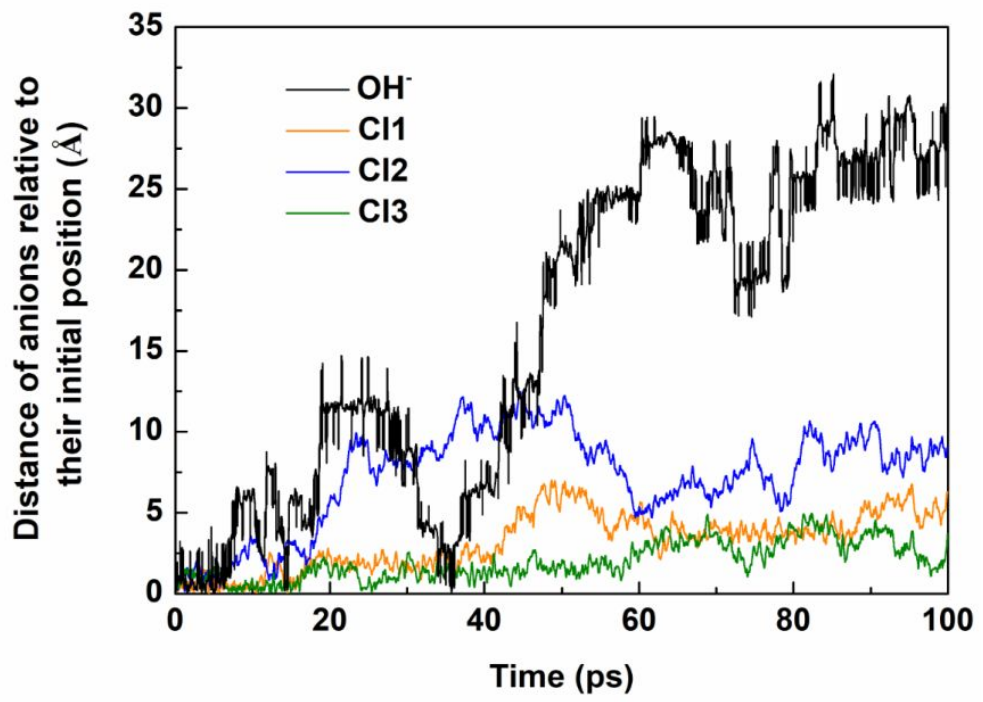

Figure S21. The distance of $\mathrm{OH}^{-}$and $\mathrm{Cl}^{-}$ions relative to their initial positions as a function of time in $\mathrm{L}$ system.

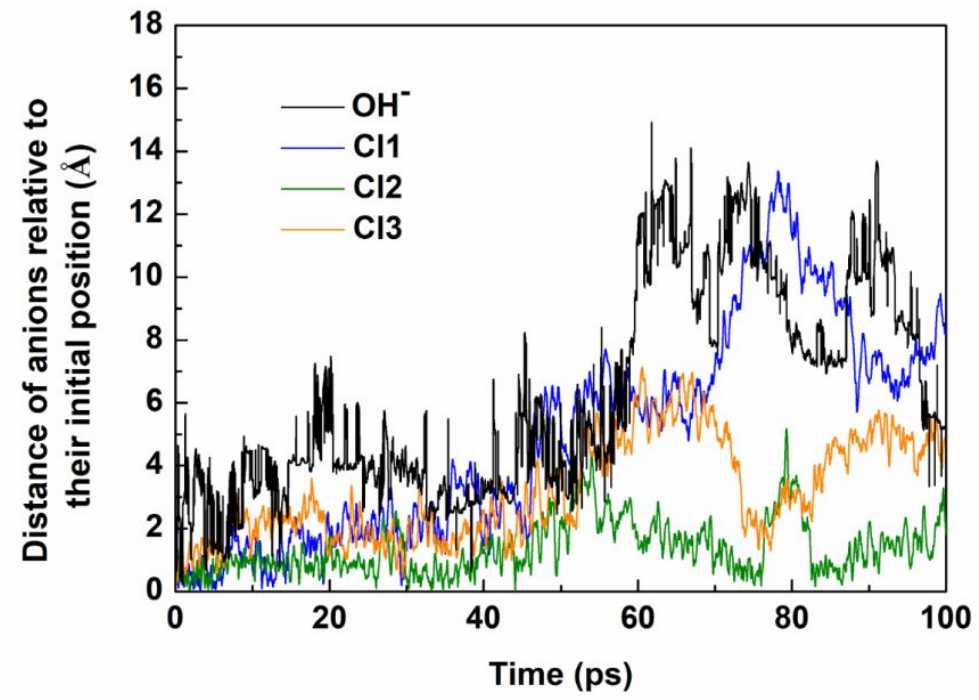

Figure S22. The distance of $\mathrm{OH}^{-}$and $\mathrm{Cl}^{-}$ions relative to their initial position as a function of time in $\mathrm{L}$ system. 
Table S1 Detailed system configurations

\begin{tabular}{cccccc}
\hline & $\mathrm{A}(\AA)$ & $\mathrm{B}(\AA)$ & $\mathrm{C}(\AA)$ & $\mathrm{N}($ water $)$ & $\mathrm{N}\left(\mathrm{Cl}^{-}\right)$ \\
\hline L system & 9.14 & 10.56 & 22.76 & 60 & 4 \\
\hline S system & 9.14 & 10.56 & 10.86 & 11 & 4 \\
\hline Bulk water & 12.45 & 12.45 & 12.45 & 64 & 0 \\
\hline 42water $+\mathrm{Cl}^{-}$ & 11.25 & 11.25 & 11.25 & 42 & 2 \\
\hline
\end{tabular}

Table S2 Hydrogen bond lifetime of L system

\begin{tabular}{lllll}
\hline $\begin{array}{l}\text { Hydrogen } \\
\text { bond }\end{array}$ & Bulk water & $\begin{array}{l}\text { IF water } v s . \\
\text { 2DLDH }\end{array}$ & $\begin{array}{l}\text { IM water } v s . \\
\text { IM water }\end{array}$ & $\begin{array}{l}\text { IF water } v s . \\
\text { IF water }\end{array}$ \\
\hline$\tau(\mathrm{ps})$ & 0.48 & 1.01 & 0.53 & 0.42 \\
\hline
\end{tabular}

Table S3 Hydrogen bond lifetime of S system

\begin{tabular}{lllll}
\hline $\begin{array}{l}\text { Hydrogen } \\
\text { bond }\end{array}$ & Bulk water & $\begin{array}{l}\text { water } v s . \\
\text { 2DLDH }\end{array}$ & $\begin{array}{l}\text { Water in } \\
\text { the same layer }\end{array}$ & $\begin{array}{l}\text { Water in } \\
\text { different layers }\end{array}$ \\
\hline$\tau(\mathrm{ps})$ & 0.48 & 0.83 & 0.36 & 0.33 \\
\hline
\end{tabular}

Table S4. Hydrogen bond analysis for different systems (hydrogen bonds connecting 2D-LDH and $\mathrm{Cl}^{-}$included)

\begin{tabular}{llllll}
\hline & $\begin{array}{l}\text { Bulk } \\
\text { water }\end{array}$ & $\begin{array}{l}\text { 42water } \\
+\mathrm{Cl}^{-}\end{array}$ & $\begin{array}{l}\text { IM region in } \\
\text { L system }\end{array}$ & $\begin{array}{l}\text { IF region in } \\
\text { L system }\end{array}$ & $\begin{array}{l}\mathrm{S} \\
\text { system }\end{array}$ \\
\hline Number of HB/water & 3.35 & 3.21 & 3.36 & 3.49 & 3.25 \\
\hline $\mathrm{H}-\mathrm{Cl}$ ratio (\%) & 0 & 5.9 & 6.3 & 9.7 & 26.0 \\
\hline $\mathrm{H}-\mathrm{OH}(2 \mathrm{DLDH})$ ratio (\%) & 0 & 0 & 0 & 28.6 & 29.0 \\
\hline
\end{tabular}

\section{References}

[S1] Luzar, A. \& Chandler, D. Hydrogen-bond kinetics in liquid water. Nature. 379, 6560 (1996). 
[S2] Bankura, A., Karmakar, A., Carnevale, V., Chandra, A. \& Klein, M. L. Structure, dynamics, and spectral diffusion of water from first-principles molecular dynamics. $J$. Phys. Chem. C 118, 29401-29411 (2014).

[S3] Cicero, G., Grossman, J. C., Schwegler, E., Gygi, F. \& Galli, G. Water confined in nanotubes and between graphene sheets: A first principle study. J. Am. Chem. Soc. 130, 1871-1878 (2008).

[S4] Sun, P., Ma, R., Bai, X., Wang, K., Zhu, H. \& Sasaki, T. Single-layer nanosheets with exceptionally high and anisotropic hydroxyl ion conductivity. Sci. Adv. 3, e1602629 (2017). 